\title{
Single-Layer Janus-Type Platinum Dichalcogenides and Their Heterostructures
}

\author{
Z. Kahraman, ${ }^{* \dagger \odot}$ A. Kandemir, ${ }^{\ddagger \odot}$ M. Yagmurcukardes, ${ }^{\S \odot}$ and H. Sahin ${ }^{\dagger, \|_{\odot}}$ \\ ${ }^{\dagger}$ Department of Photonics, ${ }^{\ddagger}$ Department of Materials Science and Engineering, and "ICTP-ECAR Eurasian Center for Advanced \\ Research, Izmir Institute of Technology, 35430 Izmir, Turkey \\ ${ }^{\S}$ Department of Physics, University of Antwerp, Groenenborgerlaan 171, B-2020 Antwerp, Belgium
}

ABSTRACT: Ultrathin two-dimensional Janus-type platinum dichalcogenide crystals formed by two different atoms at opposite surfaces are investigated by performing state-of-theart density functional theory calculations. First, it is shown that single-layer $\mathrm{PtX}_{2}$ structures (where $\mathrm{X}=\mathrm{S}$, Se, or Te) crystallize into the dynamically stable $1 \mathrm{~T}$ phase and are indirect band gap semiconductors. It is also found that the substitutional chalcogen doping in all $\mathrm{PtX}_{2}$ structures is favorable via replacement of surface atoms with a smaller chalcogen atom, and such a process leads to the formation of Janus-type platinum dichalcogenides (XPtY, where $\mathrm{X}$ and $\mathrm{Y}$ stand for $\mathrm{S}$, $\mathrm{Se}$, or Te) which are novel single-layer crystals. While all Janus structures are indirect band gap semiconductors as their binary

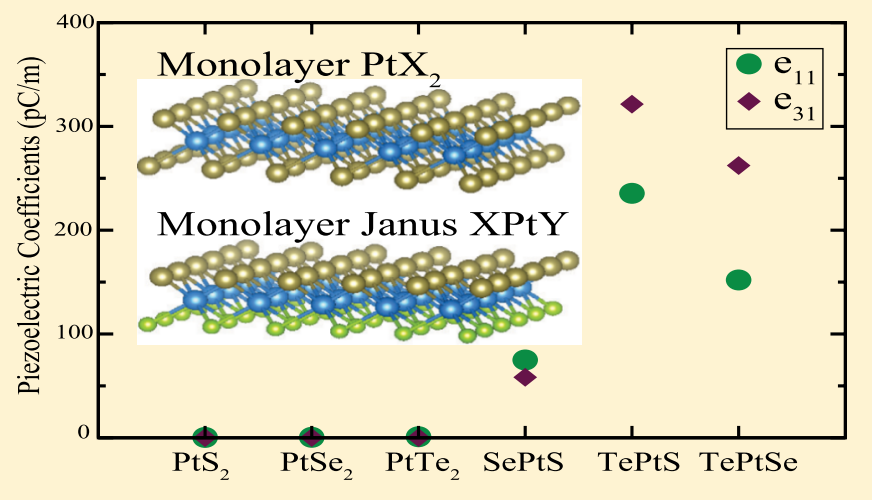
analogues, their Raman spectra show distinctive features that stem from the broken out-of-plane symmetry. In addition, it is revealed that the construction of Janus crystals enhances the piezoelectric constants of $\mathrm{PtX}_{2}$ crystals significantly both in the inplane and in the out-of-plane directions. Moreover, it is shown that vertically stacked van der Waals heterostructures of binary and ternary (Janus) platinum dichalcogenides offer a wide range of electronic features by forming bilayer heterojunctions of type-I, type-II, and type-III, respectively. Our findings reveal that Janus-type ultrathin platinum dichalcogenide crystals are quite promising materials for optoelectronic device applications.

\section{INTRODUCTION}

Interest in the field of two-dimensional (2D) single-layer materials triggered by the synthesis of graphene ${ }^{1}$ has also led to the emergence of novel ultrathin materials exhibiting remarkable optical, electronic, and mechanical properties. ${ }^{2-4}$ Apart from one-atom-thick graphene, single layers of transition metal dichalcogenides (TMDs) such as $\mathrm{MoS}_{2}, \mathrm{MoSe}_{2}, \mathrm{WS}_{2}$, and $\mathrm{WSe}_{2}$ have also been added to the library of $2 \mathrm{D}$ crystals. $^{5-20}$

Among TMDs, bulk platinum dichalcogenides $\left(\mathrm{PtX}_{2}\right.$, where $\mathrm{X}=\mathrm{S}, \mathrm{Se}$, or $\mathrm{Te}$ ) were quite demanding materials owing to their properties such as complete renewability, ${ }^{21}$ enhanced adhesion, $^{21}$ semimetallic electronic structure, ${ }^{22,23}$ high optoelectronic efficiency, ${ }^{24}$ and enhanced photo- and electrocatalytic behavior. ${ }^{25,26}$ Recently, 2D single-layer crystals of $\mathrm{PtSe}_{2}$, with high structural stability in air and high photoresponsivity, ${ }^{27-29}$ have been successfully synthesized by direct selenization of the $\mathrm{Pt}(111)$ substrate. ${ }^{30}$ In addition, theoretical studies revealed that single-layer $\mathrm{PtS}_{2}\left(\mathrm{PtSe}_{2}\right)$ is a semiconductor with a band gap of $1.6 \mathrm{eV}^{31}\left(1.2 \mathrm{eV}^{28}\right)$ having the potential for solar energy harvesting.

So far, various techniques such as the construction of both vertical and lateral heterostructures have been applied to $2 \mathrm{D}$ materials in order to combine their different properties in a material. $^{32,33}$ Although such combination is experimentally feasible, the size and quality of the heterostructure are strongly influenced by the synthesis technique, which may limit their applications. Moreover, recent studies have revealed that the synthesis of Janus-type 2D materials can be an efficient way to enrich the functionality of a $2 \mathrm{D}$ crystal because different monolayers can be combined in a single layer with strong chemical bondings. ${ }^{34}$ The fact that the two surfaces of Janus materials have different properties inspired the development of nanodevices in the fields of chemistry, biology, and physics. $^{35-40}$ Furthermore, theoretical studies have reported the energetic feasibility of Janus-type single-layer crystals such as $\mathrm{In}_{2} \mathrm{SSe}^{41} \mathrm{MoSe}_{2},{ }^{42}$ and $\mathrm{MXY}(\mathrm{M}=\mathrm{Mo}, \mathrm{W}$ and $\mathrm{X} / \mathrm{Y}=\mathrm{S}$, Se, $\mathrm{Te}) .{ }^{43,44}$ It was shown that the intrinsic out-of-plane electric field induced in a single-layer Janus crystal leads to significant Rashba spin splitting in the electronic band dispersion of WSeTe. ${ }^{43}$ In addition, synthesized Janus single layers of TMDs have been found to display higher piezoelectric performance than commonly used materials. ${ }^{44}$

In this study, motivated by the recent studies demonstrating the successful large-area chalcogen replacement in ultrathin structures, we investigate the structural, vibrational, and

Received: December 8, 2018

Revised: January 13, 2019

Published: January 22, 2019 
electronic properties of single layers of Janus-type platinum chalcogenides and their vertical heterojunctions. The paper is organized as follows: details of the computational methodology are given in Section 3. The structural, phononic, electronic, and elastic properties of the single-layer $\mathrm{PtX}_{2}$ are discussed in Section 3.1, while the properties for their Janus crystals are presented in Section 3.2. Heterojunctions of Janus structures are discussed in Section 3.3. Finally, Section 4 is devoted to the conclusion.

\section{COMPUTATIONAL DETAILS}

Density functional theory (DFT) calculations were performed using the Vienna $a b$ initio simulation package. ${ }^{45,46}$ The Perdew-Burke-Ernzerhof $(\mathrm{PBE})^{47}$ form of generalized gradient approximation was adopted to describe electron exchange and correlation. The van der Waals (vdW) correction to the PBE functional was done using the DFT long-range dispersion correction (DFT-D2) method of Grimme. ${ }^{48}$

The kinetic energy cutoff for a plane-wave basis was set to $400 \mathrm{eV}$. For the convergence criterion of self-consistent calculations in ionic relaxations, the total energy difference between the consecutive steps was taken to be $10^{-6} \mathrm{eV}$. The total force on the atoms in the primitive unit cell was reduced to a value of less than $10^{-5} \mathrm{eV} / \AA$, while the pressures on the lattice were decreased to values less than $1.0 \mathrm{kbar}$ in all directions. To eliminate interaction between the adjacent layers, at least $18 \AA$ of vacuum space was inserted. Analysis of the charge transfers in the structures was determined by using the Bader technique. ${ }^{49}$ For the band alignment calculations, the dipole corrections were taken into account in order to compensate the effect of induced out-of-plane dipole moment in Janus structures.

The cohesive energy per formula, $E_{\mathrm{Coh}}$, was calculated by using the formula; $E_{\mathrm{Coh}}=\left[n_{1} E_{1}+n_{2} E_{2}-E_{\mathrm{ML}}\right] / n_{\text {tot }}$, where $E_{1}$ and $E_{2}$ represent the energies of single isolated two atoms, respectively. $E_{\mathrm{ML}}$ stands for the total energy of the $1 \mathrm{~T}-\mathrm{PtX} \mathrm{X}_{2}$ structure and $n_{\text {tot }}, n_{1}$, and $n_{2}$ denote the total number of atoms and number of $\mathrm{Pt}$ and $\mathrm{X}$ atoms within the unit cell, respectively. The vibrational properties were calculated with Phonopy $\operatorname{code}^{50}$ that uses a finite-displacement method. Phonon eigenfrequencies in whole Brillouin Zone (BZ) and phonon eigenvectors were calculated in a 75-atom supercell. In addition, the first-order off-resonant Raman spectra were obtained by calculating the derivative of polarizability in the crystal with respect to each vibrational phonon mode at the $\Gamma$ point. $^{51}$

\section{RESULTS}

3.1. Single Layers of $\mathrm{PtX}_{2}$. 3.1.1. Structural Properties. The atomic structures of $\mathrm{PtX}_{2}$ are shown in Figure 1a. The single-layer $\mathrm{PtX}_{2}$ crystal structures exhibit hexagonal symmetry and trigonal primitive unit cell in their $1 \mathrm{~T}$ phase in which each $\mathrm{Pt}$ atom is surrounded by six $\mathrm{X}$ atoms forming an octahedral $\left[\mathrm{PtX}_{6}\right]^{4-}$ unit. For single-layer $\mathrm{PtS}_{2}, \mathrm{PtSe}_{2}$, and $\mathrm{PtTe}_{2}$, the optimized lattice parameters $(a=b)$ are calculated to be 3.57 , 3.69 , and $3.92 \AA$, respectively. As presented in Table 1 , the length parameters in the structural properties such as bond distances and geometric thickness (which is defined to be the vertical distance between uppermost and lowermost chalcogenide atoms) enlarge with the increasing atomic radius. The cohesive energy per formula in the unit cell is calculated to be $4.68 \mathrm{eV}$ for single-layer $\mathrm{PtS}_{2}$, whereas those for $\mathrm{PtSe}_{2}$ and

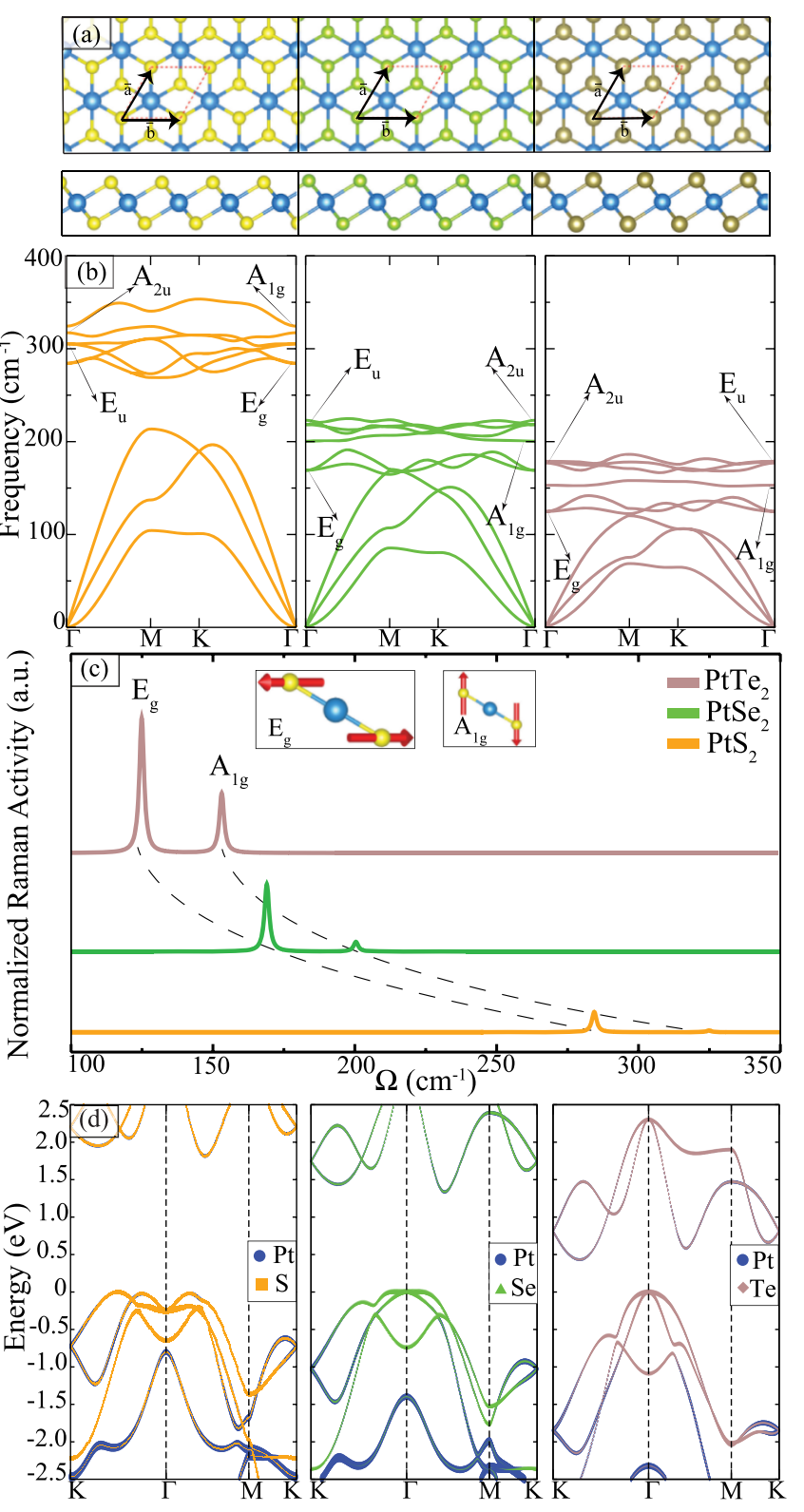

Figure 1. (a) Top side of the geometric structures of $\mathrm{PtS}_{2}, \mathrm{PtSe}_{2}$, and $\mathrm{PtTe}_{2}$. Yellow, blue, green, and brown colors show $\mathrm{S}, \mathrm{Pt}, \mathrm{Se}$, and $\mathrm{Te}$ atoms, respectively. (b) Phonon band diagrams of $\mathrm{PtS}_{2}, \mathrm{PtSe}_{2}$, and $\mathrm{PtTe}_{2}$, respectively. (c) Normalized Raman activity plot of $\mathrm{PtX}_{2}$ single layers. All Raman activities are normalized to the volume of the primitive unit cell of each crystal structure. (d) Electronic band structures of $\mathrm{PtS}_{2}, \mathrm{PtSe}_{2}$, and $\mathrm{PtTe}_{2}$.

$\mathrm{PtTe}_{2}$ are found as 4.42 and $4.28 \mathrm{eV}$, respectively. As the atomic radius of the chalcogenide atom increases from $S$ to Te, the bond length between $\mathrm{Pt}$ and $\mathrm{X}$ atoms increases, which reveals the existence of relatively weaker bonds, which means that the required energy to remove the chalcogenide atom from the structure decreases. The cohesive energies are calculated by using the formula given in Section 2 in which the energies of single isolated atoms are considered at $0 \mathrm{~K}$. In addition to cohesive energies, the heat of formation can be calculated by using the same formula. As reported in CMR data base, ${ }^{56}$ the heat of formations for $\mathrm{PtS}_{2}, \mathrm{PtSe}_{2}$, and $\mathrm{PtTe}_{2}$ are $-0.385,-0.413$, and $-0.392 \mathrm{eV} /$ atom, respectively. However, for the calculation of heat of formation, the standard state of the constituent atoms (the energy of an isolated atom 
Table 1. Calculated Parameters for Single Layers of $\mathrm{PtS}_{2}, \mathrm{PtSe}_{2}$, and $\mathrm{PtTe}_{2}$ Are as Follows: the Lattice Constants, $a=b$; the Atomic Distance between $\mathrm{Pt}$ and $\mathrm{X}$ Atoms $(\mathrm{X}=\mathrm{S}, \mathrm{Se}, \mathrm{Te}), d$; the Thickness of a Single Layer, $h$; the Cohesive Energy per Atom, $E_{\mathrm{Coh}}$; the Charge Donation from Pt to the Chalcogenide Atom, $\Delta \rho$; Work Function, $\Phi$; Total Magnetic Moment, $\mu$; Frequencies of Raman-Active Phonon Modes $\mathrm{A}_{1 \mathrm{~g}}$ and $\mathrm{E}_{\mathrm{g}}$; Intralayer Force Constant per Unit Area between Pt and X Atoms, Out-of-Plane $\left(\alpha_{\mathrm{Pt}-\mathrm{X}}^{\perp}\right)$ and In-Plane $\left(\alpha_{\mathrm{Pt}-\mathrm{X}}^{\|}\right)$Components; In-Plane and Out-of-Plane Macroscopic Static Dielectric Constants, $\epsilon_{x x}=\epsilon_{y y}$ and $\epsilon_{z z}$; In-Plane Stiffness, $C$; and Poisson's Ratio, $\nu$

\begin{tabular}{|c|c|c|c|c|c|c|c|c|c|c|c|c|c|c|c|}
\hline & $\begin{array}{c}a=b \\
(\AA)\end{array}$ & $d(\AA)$ & $h(\AA)$ & $\begin{array}{l}E_{\mathrm{Coh}} \\
(\mathrm{eV})\end{array}$ & $\begin{array}{c}\Delta \rho \\
\left(\mathrm{e}^{-}\right)\end{array}$ & $\begin{array}{c}\Phi \\
(\mathrm{eV})\end{array}$ & $\begin{array}{c}\mu \\
\left(\mu_{\mathrm{B}}\right)\end{array}$ & $\underset{\left(\mathrm{cm}^{-1}\right)}{\mathrm{A}_{1 \mathrm{~g}}}$ & $\underset{\left(\mathrm{cm}^{-1}\right)}{\mathrm{E}_{\mathrm{g}}}$ & $\begin{array}{c}\alpha_{\mathrm{Pt}-\mathrm{X}}^{\perp} \\
\left(\mathrm{N} / \mathrm{m}^{3}\right)\end{array}$ & $\begin{array}{c}\alpha_{\mathrm{Pt}-\mathrm{X}}^{\|} \\
\left(\mathrm{N} / \mathrm{m}^{3}\right)\end{array}$ & $\epsilon_{x x}=\epsilon_{y y}$ & $\begin{array}{c}\epsilon_{z z} \\
(\mathrm{~N} / \mathrm{m})\end{array}$ & C & $\nu$ \\
\hline $\mathrm{PtS}_{2}$ & 3.57 & 2.40 & 2.46 & 4.68 & 0.4 & 6.07 & 0 & 324.4 & 284.3 & $1.9 \times 10^{21}$ & $1.4 \times 10^{21}$ & 3.97 & 1.22 & 99 & 0.29 \\
\hline $\mathrm{PtSe}_{2}$ & 3.69 & 2.52 & 2.68 & 4.42 & 0.0 & 5.36 & 0 & 200.5 & 169.1 & $1.6 \times 10^{21}$ & $1.1 \times 10^{21}$ & 7.52 & 1.50 & 88 & 0.31 \\
\hline $\mathrm{PtTe}_{2}$ & 3.92 & 2.69 & 2.90 & 4.28 & -0.3 & 4.30 & 0 & 153.1 & 124.8 & $1.3 \times 10^{21}$ & $0.9 \times 10^{21}$ & 10.64 & 1.61 & 73 & 0.31 \\
\hline
\end{tabular}

is calculated at $300 \mathrm{~K}$ at $1 \mathrm{~atm}$ pressure) is taken into account. Therefore, because the standard state of each individual atom depends on their standard compounds, similar trend on cohesive energies may not be expected.

According to Bader charge analyses, formation of singlelayer $\mathrm{PtS}_{2}$ can occur by the donation of $0.4 \mathrm{e}^{-}$from each $\mathrm{Pt}$ atom to $\mathrm{S}$ atoms. In addition, while $\mathrm{PtTe}_{2}$ single layer is formed by $0.3 \mathrm{e}^{-}$transfer from each $\mathrm{Te}$ to $\mathrm{Pt}$ atom, in $\mathrm{PtSe}_{2}$ crystal structure bonds are formed without any charge transfer between $\mathrm{Pt}$ and $\mathrm{Se}$. Apparently, $\mathrm{PtSe}_{2}$ single layer has entirely covalent bonds, whereas $\mathrm{PtS}_{2}$ and $\mathrm{PtTe}_{2}$ have partially ionic bonding character.

\subsubsection{Raman Activity, Elastic Properties, and Electronic} Characteristics. The dynamical stability of the single-layer 1T$\mathrm{PtX}_{2}$ structures is verified by calculating their phonon band structures through the whole BZ. As shown in Figure 1b, all single layers of $\mathrm{PtX}_{2} \mathrm{~s}$ are free from imaginary frequencies in the whole BZ, indicating that they are dynamically stable. In the three-atom primitive unit cell, each single layer exhibits nine phonon branches three of which are acoustic and the remaining six have optical character.

While there is a large gap $\left(\sim 55 \mathrm{~cm}^{-1}\right)$ between acoustic and optical phonon branches of $\mathrm{PtS}_{2}$, for $\mathrm{PtSe}_{2}$ and $\mathrm{PtTe}_{2}$ crystals mid-frequency optical modes overlap with acoustic modes at the $\mathrm{M}$ symmetry point. As a result of the planar symmetry in the $1 \mathrm{~T}$ phase, six optical modes in $\mathrm{PtX}_{2}$ structures consist of two doubly degenerate in-plane and two nondegenerate out-ofplane phonon modes. For the single-layer $\mathrm{PtS}_{2}$, the out-ofplane modes $A_{2 u}$ and $A_{1 g}$ occur at frequencies 317.3 and 324.4 $\mathrm{cm}^{-1}$, respectively, whereas two doubly degenerate in-plane modes: $\mathrm{E}_{\mathrm{g}}$ and $\mathrm{E}_{\mathrm{u}}$ have frequencies 284.3 and $305.2 \mathrm{~cm}^{-1}$, respectively, at the $\Gamma$ point. In addition, single layers of $\mathrm{PtSe}_{2}$ and $\mathrm{PtTe}_{2}$ have $\mathrm{A}_{2 \mathrm{u}}\left(223.0\right.$ and $177.0 \mathrm{~cm}^{-1}$, respectively) and $A_{1 \mathrm{~g}}$ (200.5 and $153.1 \mathrm{~cm}^{-1}$, respectively) out-of-plane phonon modes, while they have two doubly degenerate $\mathrm{E}_{\mathrm{g}}$ (169.1 and $124.8 \mathrm{~cm}^{-1}$, respectively) and $\mathrm{E}_{\mathrm{u}}\left(218.3\right.$ and $178.8 \mathrm{~cm}^{-1}$, respectively) in-plane modes.

The group theory analysis reveals that $1 \mathrm{~T}-\mathrm{PtX}_{2}$ which belongs to the $D_{3 d}$ point group possesses three Raman-active phonon modes. The optical phonon modes, $\mathrm{E}_{\mathrm{g}}$, are attributed to the in-plane vibration of chalcogenide atoms against each other, whereas the nondegenerate $A_{1 g}$ mode represents the out-of-plane vibration of chalcogenide atoms against each other (see the inset of Figure 1c). For the two optical phonon modes, $A_{1 g}$ and $E_{g}$, the force constant per unit area can be related to the phonon frequency by the equations ${ }^{53}$

$$
\begin{aligned}
& \Omega_{\mathrm{A}_{\mathrm{lg}}}=\left(\frac{1}{2 \pi c}\right) \sqrt{\frac{2 \alpha_{\mathrm{Pt}-\mathrm{X}}^{\perp}}{\mu}} \\
& \Omega_{\mathrm{E}_{\mathrm{g}}}=\left(\frac{1}{2 \pi c}\right) \sqrt{\frac{2 \alpha_{\mathrm{Pt}-\mathrm{X}}^{\|}}{\mu}}
\end{aligned}
$$

where $\mu$ is the atomic mass per unit area and $c$ is the speed of light. Because of the contribution of only chalcogenide atoms to the vibration, the total mass per unit area is equal to $2 m_{X}$.

The calculated mass per unit area is $0.5 \times 10^{-6}, 1.1 \times 10^{-6}$, and $1.6 \times 10^{-6}\left(\mathrm{~kg} / \mathrm{m}^{2}\right)$ for $\mathrm{PtS}_{2}, \mathrm{PtSe}_{2}$, and $\mathrm{PtTe}_{2}$, respectively. As seen from Table 1, when going from $\mathrm{PtS}_{2}$ to $\mathrm{PtTe}_{2}$, the heavier the chalcogen atom, the smaller the force constants, which is directly attributed to the weaker bonds formed by the heavier chalcogenide atom. Therefore, soft crystals formed by heavier chalcogenide atoms display remarkable phonon softening in their spectrum as shown in Figure $1 \mathrm{~b}$.

In all single layers, the Raman activity of the $A_{1 g}$ mode is smaller than that of $\mathrm{E}_{\mathrm{g}}$ modes due to the relatively smaller contribution of $\epsilon_{z z}$ to the Raman tensors. ${ }^{52}$ In order to make a comparison between different single layers, the Raman activities are normalized to the volume of each primitive unit cell. As seen in Figure 1c, the Raman activity of each mode increases from $\mathrm{PtS}_{2}$ to $\mathrm{PtTe}_{2}$. Relatively larger atomic bond lengths result in larger dielectric constant which exhibits larger change of polarizability in the crystal when subjected to an incident light. Hence, the increasing Raman activity of the phonon modes from $\mathrm{PtS}_{2}$ to $\mathrm{PtTe}_{2}$ can be attributed to the increase in macroscopic static dielectric constants (see Table 1).

The linear elastic properties of 2D single-layer materials can be represented in terms of two independent constants: the inplane stiffness, $C$, and Poisson's ratio, $\nu$. For determination of linear elastic constants via energy-strain relation, a 54-atom supercell is constructed for each crystal structure. Biaxial and uniaxial strains are applied for both tensile and compressive cases. The strain parameters $\varepsilon_{\mathrm{a}}$ and $\varepsilon_{\mathrm{b}}$ are varied between \pm 0.015 with a step size of 0.005 . Then, three different sets of data are calculated: (i) $\varepsilon_{\mathrm{a}}=0$ and $\varepsilon_{\mathrm{b}}$ varying, (ii) $\varepsilon_{\mathrm{b}}=0$ and $\varepsilon_{\mathrm{a}}$ varying, and (iii) $\varepsilon_{\mathrm{a}}=\varepsilon_{\mathrm{b}}$ varying. At each configuration, the atomic positions are fully relaxed and the strain energy, $E_{S}$, is calculated by subtracting the total energy of the equilibrium state from the strained structure. Then, the calculated data are fitted to the equation: $E_{\mathrm{S}}=c_{1} \varepsilon_{\mathrm{a}}{ }^{2}+c_{2} \varepsilon_{\mathrm{b}}{ }^{2}+c_{3} \varepsilon_{\mathrm{a}} \varepsilon_{\mathrm{b}}$, ${ }^{54}$ and the coefficients, $c_{i}$, are determined. Therefore, the in-plane stiffness, $C$, the measure of the rigidity of a material under the applied external load, can be calculated by the formula: $C=\left(1 / A_{0}\right)(2 c$ $\left.-c_{3}^{2} / 2 c\right),{ }^{55}$ where $c_{1}=c_{2}$ due to the in-plane isotropy and $A_{0}$ is 
the area of the unstrained supercell. As listed in Table 1 , the $C$ values are 99, 88, and $73 \mathrm{~N} / \mathrm{m}$ from single-layer $\mathrm{PtS}_{2}$ to $\mathrm{PtTe}_{2}$. In agreement with the calculated in-plane component of intralayer force constants $\left(\alpha_{\mathrm{Pt}-\mathrm{X}}^{\|}\right)$, the larger the bond lengths, the smaller the in-plane stiffness. Decreasing trend demonstrates that as the in-plane stiffness decreases, the corresponding restoring forces in the structure become effective in a shorter range of applied strain, that is, the harmonic strain region gets smaller for soft materials. A similar trend for inplane stiffness values was reported in CMR data base $(86,71$, and $65 \mathrm{~N} / \mathrm{m}$ from single-layer $\mathrm{PtS}_{2}$ to $\left.\mathrm{PtTe}_{2}\right) .{ }^{56}$ Here, slightly different values obtained by different theoretical methodologies can be better explained via experimental measurements.

In addition, one can obtain Poisson's ratio defined as the ratio of the transverse contraction strain to the longitudinal extension strain in the direction of the stretching force. Here, Poisson's ratios for $a$ - and $b$-vectors are calculated in terms of the coefficients $c_{i}$ as $\nu_{\mathrm{a}}=\nu_{\mathrm{b}}=c_{3} / 2 c$. Poisson's ratios display small changes from single-layer $\mathrm{PtS}_{2}$ to $\mathrm{PtTe}_{2}$ (0.29 for $\mathrm{PtS}_{2}$, 0.31 for $\mathrm{PtSe}_{2}$, and $\left.0.31 \mathrm{PtTe}_{2}\right)$. When compared with wellknown TMDs, that is, 0.25 for $\mathrm{MoS}_{2},{ }^{57} 0.22$ for $\mathrm{WS}_{2},{ }^{57}$ and 0.23 for $\mathrm{MoSe}_{2},{ }^{57} \mathrm{PtX}{ }_{2}$ crystals exhibit larger responses to the applied load along the perpendicular direction. Such relatively large Poisson's ratios suggest more sensitive structural response to external loads, which can be beneficial for nanoelastic applications.

The electronic band dispersions of the single layers of $1 \mathrm{~T}$ $\mathrm{PtX}_{2}$ through the whole $\mathrm{BZ}$ are shown in Figure $1 \mathrm{~d}$. It is found that all structures are indirect band gap semiconductors. The indirect band gaps of $\mathrm{PtS}_{2}, \mathrm{PtSe}_{2}$, and $\mathrm{PtTe}_{2}$ are calculated to be $1.82,1.34$, and $0.34 \mathrm{eV}$, respectively. Therefore, the band gaps are inversely proportional to the atomic mass of their chalcogenide types. The valence band maximum (VBM) of single-layer $\mathrm{PtS}_{2}$ resides between the $K$ and $\Gamma$ points, whereas that of the other single layers is located at the $\Gamma$ point. It is seen from Figure $1 \mathrm{~d}$ that the VBM takes the shape of a sharper concave from $\mathrm{PtS}_{2}$ to $\mathrm{PtTe}_{2}$ with increasing effective mass. Conduction band minimum (CBM) shifts from within $\Gamma-M$ to within $\Gamma-K$. The CBM of single-layer $\mathrm{PtS}_{2}$ and $\mathrm{PtSe}_{2}$ resides between the $M-\Gamma$ points, whereas the $\mathrm{CBM}$ of single-layer $\mathrm{PtTe}_{2}$ resides between the $\Gamma$ and $K$ points. As seen from Figure $1 \mathrm{~d}$, the $\mathrm{CB}$ edge in between the $\Gamma$ and $K$ points becomes energetically more favorable as the radius of the chalcogenide atom increases, and it becomes CBM for single-layer $\mathrm{PtTe}_{2}$. This behavior may be attributed to the increasing lattice parameters in the structure when compared to those of $\mathrm{PtS}_{2}$ and $\mathrm{PtSe}_{2}$.

3.1.3. Chalcogen Exchange in Single-Layer PtX $X_{2}$ The exchange energies required to replace a single chalcogen atom instead of the host chalcogen atom are shown in Figure 2. The exchange energy is calculated by replacing a foreign chalcogen atom to a host chalcogen atom in a $3 \times 3 \times 1$ supercell by using the formula: $\Delta_{x c}=E_{\text {tot }}\left(\mathrm{Pt}_{9} \mathrm{X}_{18}\right)-E_{\text {tot }}\left(\mathrm{Pt}_{9} \mathrm{X}_{17} \mathrm{X}_{1}^{\prime}\right)$, where $E_{t o t}\left(\mathrm{Pt}_{9} \mathrm{X}_{18}\right)$ is the total energy of the $3 \times 3$ supercell of the bare $\mathrm{PtX}_{2}$ and $E_{\text {tot }}\left(\mathrm{Pt}_{9} \mathrm{X}_{17} \mathrm{X}_{1}^{\prime}\right)$ is the energy of the exchanged structure. Note that the chemical potential of the exchanged atom is set to be equal to its total energy in vacuum at $0 \mathrm{~K}$. By examining such exchange energy, it is possible to determine whether the replacement is obtained with or without external energy.

As seen in Figure 2, when one $S$ atom is replaced by Se or Te in a single-layer $\mathrm{PtS}_{2}$, the exchange energies are found to be 0.33 and $1.11 \mathrm{eV}$, respectively. On the other hand, if $\mathrm{S}$ or $\mathrm{Te}$

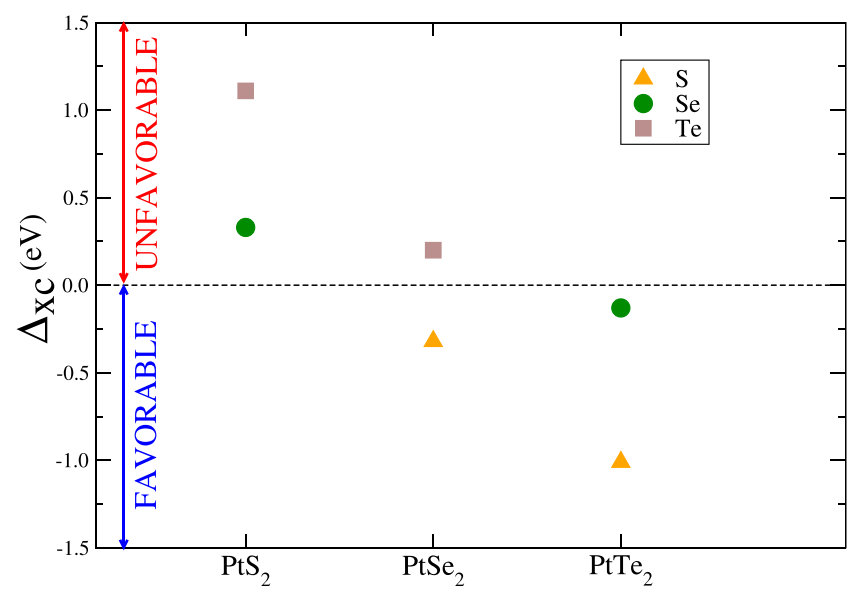

Figure 2. Exchange energy formed by the transverse process between a host and a foreign chalcogenide atom in a large-scale platinum dichalcogenide single layer.

exchanges with a Se atom in $\mathrm{PtSe}_{2}$, the required energies are calculated to be -0.32 and $0.20 \mathrm{eV}$, respectively. The minus sign indicates that the process is exothermic, whereas the plus sign means that an additional energy is required and the process is endothermic. In single-layer $\mathrm{PtTe}_{2}$, the exchange of chalcogen atoms, with $S$ or Se atoms, is found to require -0.13 and $-1.03 \mathrm{eV}$, respectively. It is clearly seen that exchanging a small chalcogen atom instead of a larger chalcogen atom is the energetically favorable process. It is found that replacing $S$ atoms instead of $\mathrm{Te}$ atoms in single layers of $\mathrm{PtTe}_{2}$ is the energetically most favorable process.

3.2. Janus-Type Single Layers of XPtY. 3.2.1. Structural Properties. Janus-type ultrathin platinum dichalcogenides are constituted completely of two different chalcogenide surfaces. After a detailed analysis of single-layer $1 \mathrm{~T}-\mathrm{PtX}_{2}$, we investigate the $1 \mathrm{~T}$ phase of Janus or, in other words, ternary platinum dichalcogenide single-layer structures, 1T-XPtY. Figure 3a shows the crystal structure of the single-layer 1T-XPtY. In a Janus structure, platinum is sandwiched between upper chalcogenide $(\mathrm{X})$ and bottom chalcogen $(\mathrm{Y})$ layers in the $1 \mathrm{~T}$ phase.

As given in Table 2, the calculated lattice parameters $(a=b)$ of ternary platinum dichalcogenides are $3.63,3.75$, and $3.81 \AA$ for SePtS, TePtS, and TePtSe, respectively. The atomic bond lengths between $\mathrm{Pt}$ atom and the upper ( $\mathrm{Se}$ ) and lower sides (S) are 2.50 and $2.43 \AA$ in the SePtS structure. On the other hand, for the TePtS structure, the Pt-Te bond length is 2.62 $\AA$, whereas that of $\mathrm{Pt}-\mathrm{S}$ is $2.57 \AA$. In single-layer TePtSe, the atomic bond lengths $\mathrm{Pt}-\mathrm{Te}$ and $\mathrm{Pt}-\mathrm{Se}$ are calculated as 2.64 and $2.57 \AA$, respectively. Moreover, the thicknesses are found to be $2.57,2.70$, and $2.80 \AA$ for SePtS, TePtS, and TePtSe, respectively. In addition, the calculated cohesive energies are 4.53, 4.40, and $4.31 \mathrm{eV}$ for SePtS, TePtS, and TePtSe, respectively. As we discussed in Section 3.1, the same trend between cohesive energies and bond lengths in the structure is found for the Janus single layers, which is a result of the existing chalcogen atoms in the Janus crystal. As mentioned for the bare $\mathrm{PtX}_{2}$ crystals, cohesive energies simply display an increasing or decreasing trend depending on the atomic mass and radius of the chalcogen atoms. For the thermodynamical stabilities of Janus crystals, the formation enthalpies at $0 \mathrm{~K}$ are calculated by using the formula: $\Delta H=E_{\text {tot }}\left(\mathrm{PtXX}^{\prime}\right)-$ $0.5 E_{\text {tot }}\left(\mathrm{PtX}_{2}^{\prime}\right)-0.5 E_{\text {tot }}\left(\mathrm{PtX}_{2}\right),{ }^{55}$ where $E_{\text {tot }}\left(\mathrm{PtXX}^{\prime}\right), E_{\text {tot }}\left(\mathrm{PtX}_{2}^{\prime}\right)$, 

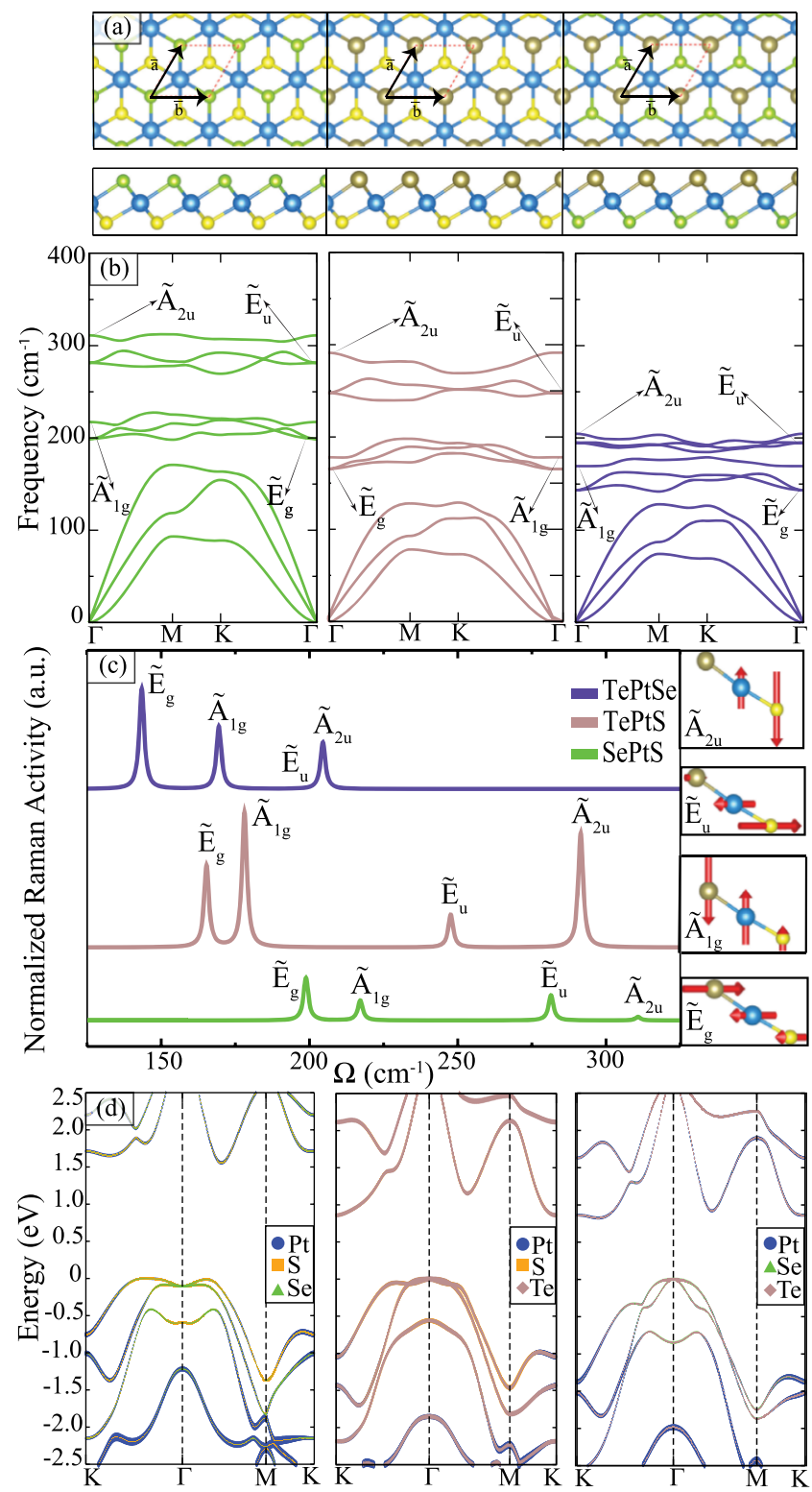

Figure 3. (a) Top and side views for the geometric structures of $\mathrm{SePtS}, \mathrm{TePtS}$, and TePtSe. Yellow, blue, green, and brown colors are for $\mathrm{S}, \mathrm{Pt}, \mathrm{Se}$, and $\mathrm{Te}$, respectively. (b) Phonon band diagrams A-like and E-like phonon branches are shown by $\tilde{\mathrm{A}}$ and $\tilde{\mathrm{E}}$, respectively. (c) Normalized Raman activity plot of Janus single layers. All Raman activities are normalized to the volume of the primitive unit cell of each crystal structure. (d) Electronic band structures of SePtS, TePtS, and $\mathrm{TePtSe}$ and the Fermi level is set to zero. and $E_{\text {tot }}\left(\mathrm{PtX}_{2}\right)$ denote the total energies of Janus and single layers of $\mathrm{PtX}_{2}$ and $\mathrm{PtX}_{2}^{\prime}$, respectively. The calculated formation enthalpies are $0.046,0.075$, and $0.243 \mathrm{eV} /$ cell for SePtS, $\mathrm{TePtSe}$, and TePtS, respectively. Our results reveal that when the mass difference between two chalcogen atoms is smaller, the formation of their Janus crystal is more feasible.

It is seen that when a small atom is placed inside the structure instead of a large atom, lattice parameters, the bond lengths, the thickness, and the cohesive energies decrease accordingly. According to Bader charge analyses, in single-layer SePtS, Pt donates $0.2 \mathrm{e}^{-}, \mathrm{S}$ receives $0.2 \mathrm{e}^{-}$, and $\mathrm{Se}$ has no charge transfer. Because charge transfers are obtained for TePtS and TePtSe, it is seen that Te donates $0.1 \mathrm{e}^{-}$to $\mathrm{Pt}$ and $0.3 \mathrm{e}^{-}$to $S$ in TePtS, whereas Te donates $0.2 \mathrm{e}^{-}$to Pt and 0.1 $\mathrm{e}^{-}$to Se in TePtSe.

3.2.2. Vibrational Properties and Raman Spectra. To verify the dynamical stability of the Janus platinum dichalcogenide structures, the vibrational properties of the $\mathrm{XPtY}$ single layers are investigated and the phonon band diagrams are shown in Figure $3 \mathrm{~b}$. Similar to their binary analogues, Janus structures are also found free from the imaginary frequencies in the whole $\mathrm{BZ}$, indicating their dynamical stability. In addition, Janus single layers exhibit three acoustical and six optical phonon branches.

As shown in Figure $3 \mathrm{~b}$, the phonon band gaps are $~ 27,36$, and $14 \mathrm{~cm}^{-1}$ for single-layer SePtS, TePtS, and TePtSe, respectively. The phonon band gaps of XPtY structures become narrower and the phonon bands soften from SePtS to TePtS and TePtSe. This can also be attributed to different atomic masses; as the mass of chalcogenide atom increases, the corresponding vibrational frequencies decrease.

Differing from the single layers of $\mathrm{PtX}_{2}$ crystals, single layers of XPtY Janus structures exhibit additional Raman-active phonon modes originating from the broken inversion symmetry. The single layers of XPtY structures exhibit six Raman-active phonon modes two of which are nondegenerate out-of-plane modes, whereas the remaining four are doubly degenerate having an in-plane vibrational character. Because the two chalcogenide atoms are different, their vibrations with respect to the Pt layer are not symmetric. Thus, depending on the atomic mass, that is, on the atomic bond lengths, the Raman activities differ from one structure to another. For instance, the phonon mode $\tilde{\mathrm{A}}_{2 \mathrm{u}}$ has the smallest Raman activity when the total $\mathrm{Pt}-\mathrm{X}$ bond length is the smallest, that is, for single-layer SePtS structure. The activity of this mode gets larger for single-layer SPtTe and decreases for single-layer SePtTe. The reason for this behavior is the vibrational character of the atoms in the phonon mode. As seen on the right panel of Figure $3 c$, one of the chalcogenide atoms is stationary and does not contribute to the vibration. In S-based $\mathrm{XPtY}$ structures, the Raman activity increases from SePtS to

Table 2. Calculated Parameters for Single Layers of SePtS, TePtS, and TePtSe Are as Follows : the Lattice Constants, $a=b$, the Atomic Distance between Pt and X Atoms $(\mathrm{X}=\mathrm{S}$, Se, Te), $d$; the Thickness of a Single Layer, $h$; the Cohesive Energy per Formula, $E_{\mathrm{Coh}}$; the Charge Donation from Pt to Chalcogens, $\Delta \rho$; Work Function, $\Phi$; Net Magnetization in the Primitive Cell, $\mu$; In-Plane and Out-of-Plane Macroscopic Static Dielectric Constants, $\epsilon_{x x}=\epsilon_{y y}$ and $\epsilon_{z z}$; In-Plane Stiffness, $C$; and Poisson's Ratio, $\nu$

\begin{tabular}{|c|c|c|c|c|c|c|c|c|c|c|c|c|c|c|c|}
\hline & $\begin{array}{c}a=b \\
(\AA)\end{array}$ & $\begin{array}{c}d_{\mathrm{Pt}-\mathrm{Se}} \\
(\AA)\end{array}$ & $\begin{array}{c}d_{\mathrm{Pt}-\mathrm{S}} \\
(\AA)\end{array}$ & $\begin{array}{c}d_{\mathrm{Pt}-\mathrm{Te}} \\
(\AA)\end{array}$ & $h(\AA)$ & $\begin{array}{l}E_{\text {Coh }} \\
(\mathrm{eV})\end{array}$ & $\begin{array}{l}\Delta \rho \\
\left(\mathrm{e}^{-}\right.\end{array}$ & $\begin{array}{c}\Phi_{\mathrm{P}-\mathrm{Se}} \\
(\mathrm{eV})\end{array}$ & $\begin{array}{l}\Phi_{\mathrm{Pt}-\mathrm{S}} \\
(\mathrm{eV})\end{array}$ & $\begin{array}{c}\Phi_{\mathrm{Pt}-\mathrm{Te}} \\
(\mathrm{eV})\end{array}$ & $\begin{array}{c}\mu \\
\left(\mu_{\mathrm{B}}\right)\end{array}$ & $\epsilon_{x x}=\epsilon_{y y}$ & $\epsilon_{z z}$ & $\begin{array}{c}C \\
(\mathrm{~N} / \mathrm{m})\end{array}$ & $\nu$ \\
\hline SePtS & 3.63 & 2.50 & 2.43 & & 2.57 & 4.53 & 0.2 & 5.31 & 6.09 & & 0 & 6.80 & 1.46 & 93 & 0.30 \\
\hline TePtS & 3.75 & & 2.57 & 2.62 & 2.70 & 4.40 & -0.1 & & 5.95 & 4.48 & 0 & 8.59 & 1.51 & 78 & 0.29 \\
\hline TePtSe & 3.81 & 2.57 & & 2.64 & 2.80 & 4.31 & -0.2 & 5.11 & & 3.05 & 0 & 9.07 & 1.55 & 76 & 0.31 \\
\hline
\end{tabular}


Table 3. Calculated Parameters for the Monolayers of $\mathrm{PtX}_{2}$ and XPtY Janus Crystals Are as Follows: the Relaxed Ion, Elastic Constants, $C_{i j}$, Piezoelectric Coefficients, $e_{i j}$, and the Corresponding Piezoelectric Strain Constants, $d_{i j}{ }^{a}$

$\begin{array}{lcccccc} & C_{11}(\mathrm{~N} / \mathrm{m}) & C_{12}(\mathrm{~N} / \mathrm{m}) & e_{11}(\mathrm{pC} / \mathrm{m}) & e_{31}(\mathrm{pC} / \mathrm{m}) & d_{11}(\mathrm{pm} / \mathrm{V}) & d_{31}(\mathrm{pm} / \mathrm{V}) \\ \mathrm{PtS}_{2} & 107.5 & 31.5 & 0.29 & 0.13 & 3.8 \times 10^{-3} & 0.9 \times 10^{-3} \\ \mathrm{PtSe}_{2} & 97.4 & 30.0 & 0.38 & 0.20 & 5.6 \times 10^{-3} & 1.5 \times 10^{-3} \\ \mathrm{PtTe}_{2} & 80.5 & 24.7 & 1.00 & 0.11 & 1.8 \times 10^{-2} & 1.3 \times 10^{-3} \\ \mathrm{SePtS} & 101.6 & 30.3 & 75.00 & 58.30 & 1.0 & 0.44 \\ \mathrm{TePtS} & 84.8 & 25.1 & 235.50 & 321.40 & 4.0 & 2.9 \\ \text { TePtSe } & 83.9 & 25.8 & 152.00 & 262.30 & 2.6 & 2.4\end{array}$

${ }^{a}$ Note that all the elastic and piezoelectric constants are renormalized by the $c$ lattice parameter in $2 \mathrm{D}$ limit.

TePtS because of the increasing mass of the stationary chalcogenide atom. By the same analogy, the $\tilde{\mathrm{E}}_{\mathrm{u}}$ mode exhibits the same behavior because it represents the in-plane vibration of $\mathrm{Pt}$ and lighter chalcogen atoms. For the $\tilde{\mathrm{A}}_{1 \mathrm{~g}}$ mode, the same trend is found because in S-based XPtY structures the vibration of $\mathrm{Se}$ and $\mathrm{Te}$ atoms dominates that of the $\mathrm{S}$ atom. When the second chalcogen atom is changed from Se to Te, the dipole between $\mathrm{Pt}$ and $\mathrm{Te}$ atoms increases because of the larger atomic bond in Pt-Te. Therefore, the Raman activity of the $\tilde{\mathrm{A}}_{\mathrm{lg}}$ increases from SePtS to TePtS. However, in single-layer TePtSe, the vibration of $\mathrm{Pt}$ and $\mathrm{Se}$ atoms has almost the same strength, whereas the vibration of $\mathrm{Te}$ dominates the motion. Finally, when we analyze the $\tilde{\mathrm{E}}_{\mathrm{g}}$ mode, it is seen that the Raman activity increases from $\mathrm{SePtS}$ to TePtSe. In all singlelayer XPtY structures, the $\mathrm{Pt}$ atom and the lighter chalcogenide atom vibrate along the same direction, whereas the heavier chalcogen atom vibrates in opposite direction. Therefore, the Raman activity increases when the bond length between $\mathrm{Pt}$ and the heavier chalcogen atom increases.

3.2.3. Elastic and Electronic Properties. Using the same methodology, the in-plane stiffness and Poisson's ratio are calculated for the Janus-type single-layer structures. As listed in Table 2, the $C$ values are 93, 78, and $76 \mathrm{~N} / \mathrm{m}$ for SePtS, TePtS, and $\mathrm{TePtSe}$, respectively. Apparently, the larger the interatomic distance, the smaller the stiffness. The decreasing trend of the in-plane stiffness also matches well with the change in cohesive energy. When these values are analyzed in terms of the individual in-plane stiffness and Poisson's ratio, in all single layers of $\mathrm{XPtY}$, the in-plane stiffness is determined by the heavier chalcogen atom. It is seen that the Te-based single layers of XPtY, TePtS, and TePtSe exhibit the same $\nu(0.31)$ values, which are dominated by the Te atom, whereas for the single layer of SePtS, $\nu$ is found to be close to that of $\mathrm{PtS}_{2}$.

The electronic band diagrams of single-layer $\mathrm{XPtY}$ are shown in Figure $3 \mathrm{~d}$. It is seen that Janus structures of SePtS, $\mathrm{TePtS}$, and $\mathrm{TePtSe}$ are indirect band gap semiconductors with band gaps of $1.56,0.86$, and $0.82 \mathrm{eV}$, respectively. In Janus structures, replacement of small atoms by larger atoms simply leads to the narrowing of the band gap. While the band structure of SePtS is determined by both and $\mathrm{S}$ and Se states, electronic bands of Te including Janus single layers are mainly composed of Te states. Compared with their binary analogues, characters of VBM and CBM in single-layer XPtY structures are significantly different. It is seen that the VBM of Janus structures shifts to the $\Gamma$ point and takes the shape of a sharper concave from SePtS to TePtSe with increasing effective mass. However, differing from the binary analogues, the CBM of Janus structures shifts from the $\Gamma-M$ to the $K$ symmetry point.

3.2.4. Piezoelectric Properties. Piezoelectricity or piezoelectric effect is known as the generating electric dipole moment as a response for the applied mechanical stress in a noncentrosymmetric material. Theoretical predictions and experimental observations have demonstrated the enhancement of piezoelectric constants in 2D limit. ${ }^{58-62}$

In the case of $1 \mathrm{~T}$-phase TMDs, because of the centrosymmetry of the structure, the piezoelectric constants are expected to be very small as compared to $1 \mathrm{H}$-phase TMDs. For the calculation of piezoelectric constants, one needs the elasticity tensor whose elements are then related to the piezoelectric coefficients, $e_{i j}$, and piezoelectric constants, $d_{i j}$, by the equation ${ }^{59}$

$$
\begin{aligned}
& d_{11}=\frac{e_{11}}{C_{11}-C_{12}} \\
& d_{31}=\frac{e_{31}}{C_{11}+C_{12}}
\end{aligned}
$$

where $C_{i j}$ stands for the second-order elastic constants. The relaxed-ion elastic constant are listed in Table 3. Because of the $D_{3 d}$ symmetry of the $1 \mathrm{~T}$ phase, $C_{11}=C_{22}$ while $C_{12}=C_{21}$, and such a symmetry also exists for piezoelectric coefficients and constants. As given by the in-plane stiffness values in Tables 1 and 2, a similar trend is found for the elastic constants, $C_{11}$ and $C_{21}$, which decrease as the atomic radius increases in $1 \mathrm{~T}-\mathrm{PtX} \mathrm{X}_{2}$ crystals.

As listed in Table 3, the calculated piezoelectric coefficients, $e_{i j}$, are negligibly small as compared to $1 \mathrm{H}-\mathrm{TMD}$ monolayers. It was experimentally observed for $1 \mathrm{H}$ monolayer $\mathrm{MoS}_{2}$ that the in-plane piezoelectric coefficient, $e_{11}$, is $294 \mathrm{pC} / \mathrm{m}^{63}$ (308 pC/ $\mathrm{m}$ in our case which reveals the reliability of the methodology). However, in the case of $1 \mathrm{~T}-\mathrm{PtX} \mathrm{X}_{2}$ crystals, the $e_{11}$ values are found to be $0.29,0.38$, and $1.00 \mathrm{pC} / \mathrm{m}$ for single-layer $\mathrm{PtS}_{2}$, $\mathrm{PtSe}_{2}$, and $\mathrm{PtTe}_{2}$, respectively, which are negligibly small as compared to monolayer $\mathrm{MoS}_{2}\left(294 \mathrm{pC} / \mathrm{m}^{63}\right)$. A similar increasing trend from $\mathrm{S}$ to Te atom was reported for $\mathrm{MX}_{2}(\mathrm{M}$ $=$ Mo or $\mathrm{W}, \mathrm{X}=\mathrm{S}, \mathrm{Se}$, or Te) structures. ${ }^{64}$ The corresponding piezoelectric constants, $d_{11}$, are listed in Table 3 which are also negligibly small as compared to 1 H-TMDs. This is a result of the in-plane centrosymmetry in $1 \mathrm{~T}$-phase crystal structures. In addition, the out-of-plane piezoelectric coefficients and the corresponding piezoelectric constants, $e_{31}$ and $d_{31}$, are also calculated and found to be comparable to those of $e_{11}$ and $d_{11}$. The finite out-of-plane piezoelectric constant arises from the noncentrosymmetric crystal structure of the $1 \mathrm{~T}$ phase in the out-of-plane direction.

When the Janus structures of $\mathrm{PtX}_{2}$ crystals are formed, due to the broken in-plane and out-of-plane symmetries, the piezoelectric constants are significantly enhanced. The in-plane piezoelectric coefficients are found to be 75.00, 235.50, and 152.00 for SePtS, TePtS, and TePtSe, respectively, and the corresponding piezoelectric constants are 1.0, 4.0, and $2.6 \mathrm{pm} /$ $\mathrm{V}$ which are at the order of $10^{-3}$ for the $\mathrm{PtX}_{2}$ structures. In 
addition to the broken in-plane symmetry in the Janus crystals, an additional out-of-plane degree of freedom allows one to have piezoelectric behavior in that direction. The calculated $e_{31}$ and $d_{31}$ values are listed in Table 3 , which follow the similar trend to what was reported for XMoY and XWY Janus crystals. ${ }^{44}$ In the Janus crystal, two chalcogenide atoms are different; thus, when an external strain is applied, different amounts of charges are accumulated on different sides of the crystal, which results in a net electric dipole moment.

3.3. Vertical vdW Heterostructures. Vertical vdW heterostructures of ultrathin materials have been the focus of interest because of the idea of Lego-type construction and combining properties of different single layers in one structure. $^{65,66}$ Because binary and ternary platinum dichalcogenides are ultrathin semiconductor materials, their vdW heterostructures and band alignments present a broad variety to achieve different electronic characters. In this section, we focus on possible vdW heterostructures and their electronic heterostructure types. Band alignments of binary and ternary (Janus type) platinum dichalcogenides are shown in Figure 4.

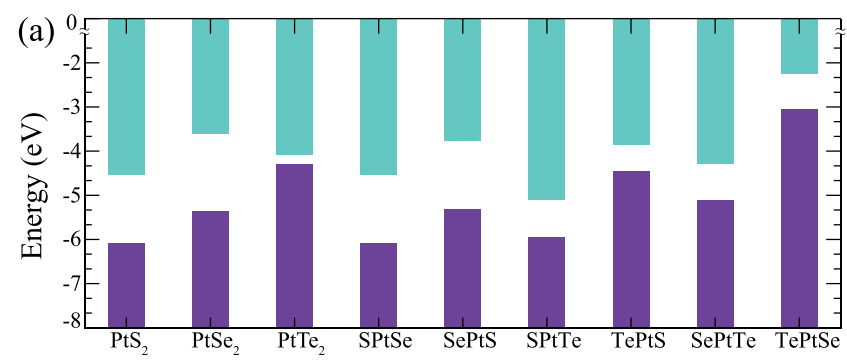

(b) \begin{tabular}{|l|l|l|l|l|l|l|l|l|}
\hline $\mathrm{PtS}_{2}$ & $\mathrm{PtSe}_{2}$ & $\mathrm{PtTe}_{2}$ & SPtSe & SePtS & SPtTe & TePtS & SePtTe & TePtSe \\
\hline
\end{tabular}

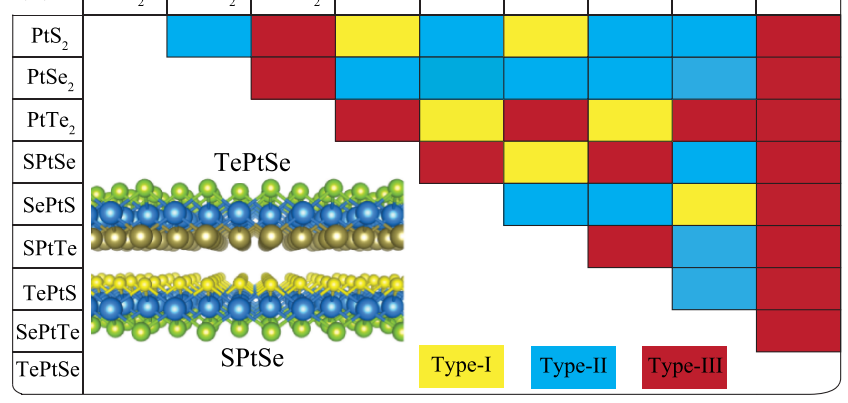

Figure 4. (a) Band alignments of binary and ternary platinum dichalcogenides. In XPtY, work function values are determined for the $\mathrm{X}$ surface and the vacuum level is set to zero. (b) Table of all possible $\mathrm{vdW}$ heterostructures types. For Janus structures, the bands are aligned according to the surface of the X-type atom. Yellow, blue, and red boxes in the table indicate type-I, -II, and -III heterostructures, respectively. Schematic representation of a heterostructure SPtSe/ $\mathrm{TePtSe}$ is also shown in the table.

Note that the dipole correction is considered in band alignment calculations to construct the exact type of alignment of the heterostructure. For this purpose, an external dipole moment in the out-of-plane direction is introduced to compensate the effect of induced dipole moment.

In type-I (straddling) heterojunctions, VBM and CBM are from the same individual layer. These types of heterostructures are common in superlattices and desirable for light-emitting applications. As seen in Figure $4 b$, it is found that the type-I vdW heterostructures can be formed by $\mathrm{PtS}_{2} / \mathrm{SPtSe}, \mathrm{PtS}_{2} /$ SPtTe, $\mathrm{PtTe}_{2} / \mathrm{SePtS}, \mathrm{PtTe}_{2} / \mathrm{TePtS}, \mathrm{SePtS} / \mathrm{SePtTe}$, and SPtSe/ SPtTe.
If both VBM and CBM of one structure are lower than the VBM and CBM of the other structure, electrons are confined in one structure, whereas holes are confined in the other structure. In this case, the heterostructure type is called as type-II or staggered-type heterojunction. Owing to separation of electrons and holes to different layers, the lifetime of radiative recombinations is increased; thus, these types of heterostructures can be used for photocatalytic, photovoltaics, and photodetection applications. $\mathrm{PtS}_{2} / \mathrm{PtSe}_{2}, \mathrm{PtS}_{2} / \mathrm{SePtS}$, $\mathrm{PtS}_{2} / \mathrm{SePtTe}, \mathrm{PtS}_{2} / \mathrm{TePtS}, \mathrm{PtSe}_{2} / \mathrm{SePtS}, \mathrm{PtSe}_{2} / \mathrm{SePtTe}$, $\mathrm{PtSe}_{2} / \mathrm{SPtSe}, \mathrm{PtSe}_{2} / \mathrm{SPtTe}, \mathrm{PtSe}_{2} / \mathrm{TePtS}, \mathrm{SePtS} / \mathrm{SPtTe}$, $\mathrm{SePtS} / \mathrm{TePtS}, \mathrm{SePtTe} / \mathrm{TePtS}, \mathrm{SPtSe} / \mathrm{SePtTe}$, and $\mathrm{SPtTe} /$ SePtTe vdW heterostructures are found to form type-II heterojunctions.

In a type-III (broken) heterojunction, the CBM of one structure lies below the VBM of the other one. Such heterojunctions not only allow semimetal-to-semiconductor transitions but also lead to the formation of interface excitons and coexistence of electrons and holes. We found that $\mathrm{PtS}_{2} /$ $\mathrm{PtTe}_{2}, \mathrm{PtS}_{2} / \mathrm{TePtSe}, \mathrm{PtSe}_{2} / \mathrm{PtTe}_{2}, \mathrm{PtSe}_{2} / \mathrm{TePtSe}_{\mathrm{PtTe}} /$ SePtTe, PtTe $2 / S P t S e, P_{T} e_{2} / S P t T e, P_{T} e_{2} / T_{e P t S e}, \mathrm{SePtS} /$ TePtSe, SPtSe/TePtS, SPtSe/SePtS, SPtSe/TePtSe, SPtTe/ TePtS, SPtTe/TePtSe, TePtS/TePtSe, and TePtSe/SePtTe are type-III heterojunctions.

\section{CONCLUSIONS}

In the present work, the structural, electronic, and vibrational properties of the single layers of $\mathrm{PtX}_{2}$ and Janus-type $\mathrm{XPtY}$ (where $\mathrm{X} / \mathrm{Y}=\mathrm{S}$, Se, Te) were investigated by performing $\mathrm{ab}$ initio calculations. It was shown that single-layer $\mathrm{PtX}_{2}$ structures are dynamically stable structures in the $1 \mathrm{~T}$ phase and display three characteristic Raman-active phonon modes at the $\Gamma$ point that can be observed by Raman measurements.

Furthermore, it was found that formation of Janus-type platinum dichalcogenide crystals having two different atoms at opposite surfaces is possible via chalcogen exchange and all $\mathrm{XPtY}$ are dynamically stable. In addition, in their Raman spectrum, Janus XPtY structures exhibit additional three Raman-active modes stemming from the broken out-of-plane symmetry. Electronically, similar to their binary analogues, all Janus-type platinum chalcogenide structures were calculated to be indirect band gap semiconductor. Moreover, the construction of Janus crystals enhances the piezoelectric constants of $\mathrm{PtX}_{2}$ crystals significantly and adds a new degree of freedom for piezoelectricity in the out-of-plane direction. The vdW heterostructures of binary and ternary platinum dichalcogenides were also investigated. It was found that 36 possible vdW heterostructures create ultrathin heterojunctions of type-I, -II, and -III. Our study reveals that Janus-type platinum dichalcogenide crystals are novel stable materials having a wide range of electronic characters.

\section{AUTHOR INFORMATION}

\section{Corresponding Author}

*E-mail: zeynepkahraman@iyte.edu.tr. ORCID $\odot$

Z. Kahraman: 0000-0002-1729-0812

A. Kandemir: 0000-0001-9813-6421

M. Yagmurcukardes: 0000-0002-1416-7990

H. Sahin: 0000-0002-6189-6707

Notes

The authors declare no competing financial interest. 


\section{ACKNOWLEDGMENTS}

Computational resources were provided by TUBITAK ULAKBIM, High Performance and Grid Computing Center (TR-Grid e-Infrastructure). H.S. and Z.K. acknowledge financial support from the TUBITAK under the project number 117F095. This work is supported by the Flemish Science Foundation (FWO-Vl) by a post-doctoral fellowship (M.Y.). H.S. acknowledges support from Türkiye Bilimler Akademisi-Turkish Academy of Sciences under the GEBIP program.

\section{REFERENCES}

(1) Novoselov, K. S.; Geim, A. K.; Morozov, S. V.; Jiang, D.; Zhang, Y.; Dubonos, S. V.; Grigorieva, I. V.; Firsov, A. A. Electric Field Effect in Atomically Thin Carbon Films. Science 2004, 306, 666-669.

(2) Mas-Ballesté, R.; Gómez-Navarro, C.; Gómez-Herrero, J.; Zamora, F. 2D materials: to graphene and beyond. Nanoscale 2011, 3, 20-30.

(3) Wang, Q. H.; Kalantar-Zadeh, K.; Kis, A.; Coleman, J. N.; Strano, M. S. Electronics and optoelectronics of two-dimensional transition metal dichalcogenides. Nat. Nanotechnol. 2012, 7, 699-712.

(4) Kim, K. K.; Hsu, A.; Jia, X.; Kim, S. M.; Shi, Y.; Hofmann, M.; Nezich, D.; Rodriguez-Nieva, J. F.; Dresselhaus, M.; Palacios, T.; Kong, J. Synthesis of Monolayer Hexagonal Boron Nitride on Cu Foil Using Chemical Vapor Deposition. Nano Lett. 2012, 12, 161-166.

(5) Mak, K. F.; Lee, C.; Hone, J.; Shan, J.; Heinz, T. F. Atomically Thin $\mathrm{MoS}_{2}$ : A New Direct-Gap Semiconductor. Phys. Rev. Lett. 2010, $105,136805$.

(6) Ramakrishna Matte, H. S. S.; Gomathi, A.; Manna, A. K.; Late, D. J.; Datta, R.; Pati, S. K.; Rao, C. N. R. $\mathrm{MoS}_{2}$ and $\mathrm{WS}_{2}$ Analogues of Graphene. Angew. Chem., Int. Ed. 2010, 49, 4059-4062.

(7) Lee, C.; Yan, H.; Brus, L. E.; Heinz, T. F.; Hone, J.; Ryu, S. Anomalous Lattice Vibrations of Single- and Few-Layer $\mathrm{MoS}_{2}$. ACS Nano 2010, 4, 2695-2700.

(8) Splendiani, A.; Sun, L.; Zhang, Y.; Li, T.; Kim, J.; Chim, C.-Y.; Galli, G.; Wang, F. Emerging Photoluminescence in Monolayer $\mathrm{MoS}_{2}$. Nano Lett. 2010, 10, 1271-1275.

(9) Coleman, J. N.; Lotya, M.; O’Neill, A.; Bergin, S. D.; King, P. J.; Khan, U.; Young, K.; Gaucher, A.; De, S.; Smith, R. J.; Shvets, I. V.; Arora, S. K.; Stanton, G.; Kim, H.-Y.; Lee, K.; Kim, G. T.; Duesberg, G. S.; Hallam, T.; Boland, J. J.; Wang, J. J.; Donegan, J. F.; Grunlan, J. C.; Moriarty, G.; Shmeliov, A.; Nicholls, R. J.; Perkins, J. M.; Grieveson, E. M.; Theuwissen, K.; McComb, D. W.; Nellist, P. D.; Nicolosi, V. Two-Dimensional Nanosheets Produced by Liquid Exfoliation of Layered Materials. Science 2011, 331, 568-571.

(10) Ataca, C.; Şahin, H.; Ciraci, S. Stable, Single-Layer $\mathrm{MX}_{2}$ Transition-Metal Oxides and Dichalcogenides in a Honeycomb-Like Structure. J. Phys. Chem. C 2012, 116, 8983-8999.

(11) Yin, Z.; Li, H.; Li, H.; Jiang, L.; Shi, Y.; Sun, Y.; Lu, G.; Zhang, Q.; Chen, X.; Zhang, H. Single-Layer $\mathrm{MoS}_{2}$ Phototransistors. ACS Nano 2012, 6, 74-80.

(12) Chhowalla, M.; Shin, H. S.; Eda, G.; Li, L.-J.; Loh, K. P.; Zhang, $\mathrm{H}$. The chemistry of two-dimensional layered transition metal dichalcogenide nanosheets. Nat. Chem. 2013, 5, 263-275.

(13) Zhang, Y.; Chang, T.-R.; Zhou, B.; Cui, Y.-T.; Yan, H.; Liu, Z.; Schmitt, F.; Lee, J.; Moore, R.; Chen, Y.; Lin, H.; Jeng, H.-T.; Mo, S.K.; Hussain, Z.; Bansil, A.; Shen, Z.-X. Direct observation of the transition from indirect to direct bandgap in atomically tin epitaxial $\mathrm{MoSe}_{2}$. Nat. Nanotechnol. 2014, 9, 111-115.

(14) Lu, X.; Utama, M. I. B.; Lin, J.; Gong, X.; Zhang, J.; Zhao, Y.; Pantelides, S. T.; Wang, J.; Dong, Z.; Liu, Z.; Zhou, W.; Xiong, Q. Large-Area Synthesis of Monolayer and Few-Layer $\mathrm{MoSe}_{2}$ Films on $\mathrm{SiO}_{2}$ Substrates. Nano Lett. 2014, 14, 2419-2425.

(15) Huo, N.; Kang, J.; Wei, Z.; Li, S.-S.; Li, J.; Wei, S.-H. Novel and Enhanced Optoelectronic Performances of Multilayer $\mathrm{MoS}_{2}-\mathrm{WS}_{2}$ Heterostructure Transistors. Adv. Funct. Mater. 2014, 24, 7025-7031.
(16) Chernikov, A.; van der Zande, A. M.; Hill, H. M.; Rigosi, A. F.; Velauthapillai, A.; Hone, J.; Heinz, T. F. Electrical Tuning of Exciton Binding Energies in Monolayer $\mathrm{WS}_{2}$. Phys. Rev. Lett. 2015, 115, 126802 .

(17) Koperski, M.; Nogajewski, K.; Arora, A.; Cherkez, V.; Mallet, P.; Veuillen, J.-Y.; Marcus, J.; Kossacki, P.; Potemski, M. Single photon emitters in exfoliated $\mathrm{WSe}_{2}$ structures. Nat. Nanotechnol. 2015, 10, 503-506.

(18) Esfahani, D. N.; Leenaerts, O.; Sahin, H.; Partoens, B.; Peeters, F. M. Structural Transitions in Monolayer $\mathrm{MoS}_{2}$ by Lithium Adsorption. J. Phys. Chem. C 2015, 119, 10602-10609.

(19) Island, J. O.; Kuc, A.; Diependaal, E. H.; Bratschitsch, R.; van der Zant, H. S. J.; Heine, T.; Castellanos-Gomez, A. Precise and reversible band gap tuning in single-layer $\mathrm{MoSe}_{2}$ by uniaxial strain. Nanoscale 2016, 8, 2589-2593.

(20) Chuang, H.-J.; Chamlagain, B.; Koehler, M.; Perera, M. M.; Yan, J.; Mandrus, D.; Tománek, D.; Zhou, Z. Low-Resistance 2D/2D Ohmic Contacts: A Universal Approach to High-Performance WSe ${ }_{2}$, $\mathrm{MoS}_{2}$, and $\mathrm{MoSe}_{2}$ Transistors. Nano Lett. 2016, 16, 1896-1902.

(21) Soled, S.; Wold, A.; Gorochov, O. Crystal growth and characterization of several platinum sulfoselenides. Mater. Res. Bull. 1976, 11, 927-932.

(22) Guo, G. Y.; Liang, W. Y. The electronic structures of platinum dichalcogenides: $\mathrm{PtS}_{2}, \mathrm{PtSe}_{2}$ and $\mathrm{PtTe}_{2}$. J. Phys. C: Solid State Phys. 1986, 19, 995-1008.

(23) Kliche, G. Far-infrared and X-ray investigations of the mixed platinum dichalcogenides $\mathrm{PtS}_{2-\mathrm{x}} \mathrm{Se}_{\mathrm{x}}, \mathrm{PtSe}_{2-\mathrm{x}} \mathrm{Te}_{\mathrm{x}}$ and $\mathrm{PtS}_{2-\mathrm{x}} \mathrm{Te}_{\mathrm{x}}$. J. Solid State Chem. 1985, 56, 26-31.

(24) Yeo, J. S. L.; Vittal, J. J.; Henderson, W.; Andy Hor, T. S. Exploring the Lewis basicity of the metalloligand $\left[\mathrm{Pt}_{2}(\hat{\mathrm{I}} 1-\right.$ $\left.\mathrm{Se})_{2}\left(\mathrm{PPh}_{3}\right)_{4}\right]$ on metal substrates by electrospray mass spectrometry. Synthesis, characterization and structural studies of new platinum selenido phosphine complexes containing the $\mathrm{Pt}_{2} \mathrm{Se}_{2}$ core. J. Chem. Soc., Dalton Trans. 2002, 328-336.

(25) Chia, X.; Adriano, A.; Lazar, P.; Sofer, Z.; Luxa, J.; Pumera, M. Layered Platinum Dichalcogenides $\left(\mathrm{PtS}_{2}, \mathrm{PtSe}_{2}\right.$, and $\left.\mathrm{PtTe}_{2}\right)$ Electrocatalysis: Monotonic Dependence on the Chalcogen Size. Adv. Funct. Mater. 2016, 26, 4306-4318.

(26) Lu, Q.; Yu, Y.; Ma, Q.; Chen, B.; Zhang, H. 2D TransitionMetal-Dichalcogenide-Nanosheet-Based Composites for Photocatalytic and Electrocatalytic Hydrogen Evolution Reactions. Adv. Funct. Mater. 2016, 28, 1917-1933.

(27) Zhao, Y.; Qiao, J.; Yu, Z.; Yu, P.; Xu, K.; Lau, S. P.; Zhou, W.; Liu, Z.; Wang, X.; Ji, W.; Chai, Y. High-Electron-Mobility and AirStable 2D Layered $\mathrm{PtSe}_{2}$ FETs. Adv. Mater. 2017, 29, 1604230.

(28) Wang, Y.; Li, L.; Yao, W.; Song, S.; Sun, J. T.; Pan, J.; Ren, X.; Li, C.; Okunishi, E.; Wang, Y.-Q.; Wang, E.; Shao, Y.; Zhang, Y. Y.; Yang, H.-t.; Schwier, E. F.; Iwasawa, H.; Shimada, K.; Taniguchi, M.; Cheng, Z.; Zhou, S.; Du, S.; Pennycook, S. J.; Pantelides, S. T.; Gao, H.-J. Monolayer $\mathrm{PtSe}_{2}$, a New Semiconducting Transition-MetalDichalcogenide, Epitaxially Grown by Direct Selenization of Pt. Nano Lett. 2015, 15, 4013-4018.

(29) Yim, C.; Lee, K.; McEvoy, N.; O’Brien, M.; Riazimehr, S.; Berner, N. C.; Cullen, C. P.; Kotakoski, J.; Meyer, J. C.; Lemme, M. C.; Duesberg, G. S. High-Performance Hybrid Electronic Devices from Layered $\mathrm{PtSe}_{2}$ Films Grown at Low Temperature. ACS Nano 2016, 10, 9550-9558.

(30) Sajjad, M.; Singh, N.; Schwingenschlögl, U. Strongly bound excitons in monolayer $\mathrm{PtS}_{2}$ and $\mathrm{PtSe}_{2}$. Appl. Phys. Lett. 2018, 112, 043101.

(31) Zhao, Y.; Qiao, J.; Yu, P.; Hu, Z.; Lin, Z.; Lau, S. P.; Liu, Z.; Ji, W.; Chai, Y. Extraordinarily Strong Interlayer Interaction in $2 \mathrm{D}$ Layered $\mathrm{PtS}_{2}$. Adv. Mater. 2016, 28, 2399-2407.

(32) Stan, G. B.-M.; Toroker, M. C. Lateral Chemical Bonding in Two-Dimensional Transition-Metal Dichalcogenide Metal/Semiconductor Heterostructures. J. Phys. Chem. C 2018, 122, 5401-5410.

(33) Woods, J. M.; Xie, Y.; Liu, W.; Liu, Y.; Wang, H.; Cha, J. J.; Cha, J. J. One-Step Synthesis of $\mathrm{MoS}_{2} / \mathrm{WS}_{2}$ Layered Heterostructures 
and Catalytic Activity of Defective Transition Metal Dichalcogenide Films. ACS Nano 2016, 10, 2004-2009.

(34) Lu, A.-Y.; Zhu, H.; Xiao, J.; Chuu, C.-P.; Han, Y.; Chiu, M.-H.; Cheng, C.-C.; Yang, C.-W.; Wei, K.-H.; Yang, Y.; Wang, Y.; Sokaras, D.; Nordlund, D.; Yang, P.; Muller, D. A.; Chou, M.-Y.; Zhang, X.; Li, L.-J. Janus monolayers of transition metal dichalcogenides. Nat. Nanotechnol. 2017, 12, 744.

(35) Zhang, J.; Grzybowski, B. A.; Granick, S. Janus Particle Synthesis, Assembly, and Application. Langmuir 2017, 33, 69646977.

(36) Zhao, G.; Pumera, M. Geometric asymmetry driven Janus micromotors. Nanoscale 2014, 6, 11177-11180.

(37) Wu, Y.; Lin, X.; Wu, Z.; Möhwald, H.; He, Q. Self-Propelled Polymer Multilayer Janus Capsules for Effective Drug Delivery and Light-Triggered Release. ACS Appl. Mater. Interfaces 2014, 6, 1047610481.

(38) Ma, Q.; Yu, W.; Dong, X.; Wang, J.; Liu, G. Janus nanobelts: fabrication, structure and enhanced magnetic-fluorescent bifunctional performance. Nanoscale 2014, 6, 2945-2952.

(39) Riis-Jensen, A. C.; Pandey, M.; Thygesen, K. S. Efficient Charge Separation in 2D Janus van der Waals Structures with Built-in Electric Fields and Intrinsic p-n Doping. J. Phys. Chem. C 2018, 122, 2452024526.

(40) Palsgaard, M.; Gunst, T.; Markussen, T.; Thygesen, K. S.; Brandbyge, M. Stacked Janus Device Concepts: Abrupt pn-Junctions and Cross-Plane Channels. Nano Lett. 2018, 18, 7275-7281.

(41) Kandemir, A.; Sahin, H. Janus single layers of $\operatorname{In}_{2} \mathrm{SSe}$ : A firstprinciples study. Phys. Rev. B 2018, 97, 155410.

(42) Zhang, J.; Jia, S.; Kholmanov, I.; Dong, L.; Er, D.; Chen, W.; Guo, H.; Jin, Z.; Shenoy, V. B.; Shi, L.; Lou, J. Janus Monolayer Transition-Metal Dichalcogenides. ACS Nano 2017, 11, 8192-8198.

(43) Cheng, Y. C.; Zhu, Z. Y.; Tahir, M.; Schwingenschlögl, U. Spinorbit-induced spin splittings in polar transition metal dichalcogenide monolayers. Europhys. Lett. 2013, 102, 57001.

(44) Dong, L.; Lou, J.; Shenoy, V. B. Large In-Plane and Vertical Piezoelectricity in Janus Transition Metal Dichalchogenides. ACS Nano 2017, 11, 8242-8248.

(45) Kresse, G.; Hafner, J. Ab initiomolecular dynamics for liquid metals. Phys. Rev. B: Condens. Matter Mater. Phys. 1993, 47, 558.

(46) Kresse, G.; Furthmüller, J. Efficient iterative schemes forab initiototal-energy calculations using a plane-wave basis set. Phys. Rev. B: Condens. Matter Mater. Phys. 1996, 54, 11169.

(47) Perdew, J. P.; Burke, K.; Ernzerhof, M. Generalized Gradient Approximation Made Simple. Phys. Rev. Lett. 1996, 77, 3865.

(48) Grimme, S. Semiempirical GGA-type density functional constructed with a long-range dispersion correction. J. Comput. Chem. 2006, 27, 1787-1799.

(49) Henkelman, G.; Arnaldsson, A.; Jónsson, H. A fast and robust algorithm for Bader decomposition of charge density. Comput. Mater. Sci. 2006, 36, 354-360.

(50) Togo, A.; Oba, F.; Tanaka, I. First-principles calculations of the ferroelastic transition between rutile-type and $\mathrm{CaCl}_{2}$-type $\mathrm{SiO}_{2}$ at high pressures. Phys. Rev. B: Condens. Matter Mater. Phys. 2008, 78, 134106.

(51) Fonari, A.; Stauffer, S. https://github.com/raman-sc/VASP/, 2014.

(52) Yagmurcukardes, M.; Bacaksiz, C.; Unsal, E.; Akbali, B.; Senger, R. T.; Sahin, H. Strain mapping in single-layer two-dimensional crystals via Raman activity. Phys. Rev. B 2018, 97, 115427.

(53) Zhang, X.; Han, W. P.; Wu, J. B.; Milana, S.; Lu, Y.; Li, Q. Q.; Ferrari, A. C.; Tan, P. H. Raman spectroscopy of shear and layer breathing modes in multilayer $\mathrm{MoS}_{2}$. Phys. Rev. B: Condens. Matter Mater. Phys. 2013, 87, 115413.

(54) Nye, J. F. Physical Properties of Crystals; Clarendon Press: Oxford, 1985

(55) Kang, J.; Sahin, H.; Peeters, F. M. Mechanical properties of monolayer sulphides: a comparative study between $\mathrm{MoS}_{2}, \mathrm{HfS}_{2}$ and $\mathrm{TiS}_{3}$. Phys. Chem. Chem. Phys. 2015, 17, 27742-27749.
(56) Haastrup, S.; Strange, M.; Pandey, M.; Deilmann, T.; Schmidt, P. S.; Hinsche, N. F.; Gjerding, M. N.; Torelli, D.; Larsen, P. M.; RiisJensen, A. C.; et al. The Computational 2D Materials Database: highthroughput modeling and discovery of atomically thin crystals. $2 D$ Mater. 2018, 5, 042002.

(57) Guzman, D. M.; Strachan, A. Role of strain on electronic and mechanical response of semiconducting transition-metal dichalcogenide monolayers: An ab-initio study. J. Appl. Phys. 2014, 115, 243701.

(58) Blonsky, M. N.; Zhuang, H. L.; Singh, A. K.; Hennig, R. G. Ab Initio Prediction of Piezoelectricity in Two-Dimensional Materials. ACS Nano 2015, 9, 9885-9891.

(59) Alyörük, M. M.; Aierken, Y.; Çakır, D.; Peeters, F. M.; Sevik, C. Promising piezoelectric performance of single layer transition-metal dichalcogenides and dioxides. J. Phys. Chem. C 2015, 119, 2323123237.

(60) Fei, R.; Li, W.; Li, J.; Yang, L. Giant Piezoelectricity of Monolayer Group IV Monochalcogenides: SnSe, SnS, GeSe, and GeS. Appl. Phys. Lett. 2015, 107, 173104.

(61) Li, W.; Li, J. Piezoelectricity in Two-Dimensional Group-III Monochalcogenides. Nano Res. 2015, 8, 3796-3802.

(62) Gomes, L. C.; Carvalho, A.; Castro Neto, A. H. Enhanced Piezoelectricity and Modified Dielectric Screening of Two Dimensional Group-IV Monochalcogenides. Phys. Rev. B: Condens. Matter Mater. Phys. 2015, 92, 214103.

(63) Zhu, H.; Wang, Y.; Xiao, J.; Liu, M.; Xiong, S.; Wong, Z. J.; Ye, Z.; Ye, Y.; Yin, X.; Zhang, X. Observation of Piezoelectricity in FreeStanding Monolayer $\mathrm{MoS}_{2}$. Nat. Nanotechnol. 2014, 10, 151-155.

(64) Duerloo, K. A. N.; Ong, M. T.; Reed, E. J. Intrinsic Piezoelectricity in Two-Dimensional Materials. J. Phys. Chem. Lett. 2012, 3, 2871-2876.

(65) Geim, A. K.; Grigorieva, I. V. Van der Waals heterostructures. Nature 2013, 499, 419-425.

(66) Novoselov, K. S.; Mishchenko, A.; Carvalho, A.; Castro Neto, A. H. 2D materials and van der Waals heterostructures. Science 2016, 353, aac9439. 